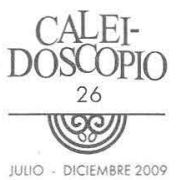

\title{
Un pueblo con vocación textil: Trazos históricos de Villa Hidalgo, Jalisco $^{1}$
}

Villa Hidalgo, Jalisco, es una población cuyos habitantes, sin contar con antecedentes industriales, pasaron de dedicarse a actividades agrícolas a emprender micronegocios de producción y venta de prendas de vestir, ocupación de la cual vive la mayoría de los villahidalguenses desde hace al menos tres décadas hasta la actualidad. En el transcurso de los años, esa población ha ganado una fama de centro del vestir que constituye un interesante objeto de estudio.

Por ello, en este artículo se presentan algunos trazos históricos del surgimiento y desarrollo de la industria del vestido en Villa Hidalgo, Jalisco. Lo que se pretende es mostrar elementos que ayuden a entender cómo ha llegado a ser una actividad tradicional de rápida expansión y con una gran capacidad de ocupar personal, a pesar de que sus características productivas son de tipo tradicional familiar, poca organización y baja productividad.

A continuación se presentará, en primer lugar, la ubicación de Villa Hidalgo en el contexto regional de Los Altos de Jalisco, y

Este artículo se basa en los resultados de entrevistas a dueños y empleados de tiendas y talleres de ropa de Villa Hidalgo, Jalisco, realizadas para el trabajo de tesis doctoral de la coautora.

2 Profesora investigadora de la Universidad Panamericana campus Aguascalientes, (cgil@up.edu. $\underline{\mathrm{mx}})$

3 Profesor investigador de la Universidad Autónoma de Aguascalientes (ommaza@correo.uaa. $\mathrm{mx})$. 
posteriormente la cronología del surgimiento y desarrollo de la industria del vestido en ese lugar.

\section{ViLla Hidalgo en SU CONTEXTO REGIONAL}

Villa Hidalgo nació en 1738. Su nombre primitivo fue "Paso de Carretas", pues era paraje de quienes iban y venían de Aguascalientes. Posteriormente se llamó de diversas maneras: "Paso Esparza", luego "Paso de la Santísima Trinidad de los Sotos" y "Paso de Sotos". Desde 1869 fue reconocido como municipio, perteneciente al onceavo cantón de Teocaltiche. El 30 de mayo de 1922 se le dio el nombre de Villa Hidalgo, en honor a Miguel Hidalgo y Costilla, quien al parecer pernoctó en el poblado después del desastre en Puente de Calderón (Botello et al, 1987).

Hasta los años sesentas, Villa Hidalgo "era un pueblo de burros, ni siquiera bicicletero (...) en las calles circulaban más bien puros burros o caballos, porque no había carros (...) no había luz eléctrica (...), las casas se alumbraban con aparatos de petróleo (...) y todo mundo tenía en el corral sus vacas, sus puercos, sus gallinas, todos sus animalitos de donde se mantenía la gente (...) del campo, había unos pocos comerciantes y agricultores muchos" (A4).

Actualmente, por el uso del suelo, se podría considerar a Villa Hidalgo como población rural, pero por el número de habitantes; ${ }^{4}$ su bajo índice de marginalidad y su conexión con Aguascalientes, ${ }^{5}$ es posible calificarlo como asentamiento urbano. ${ }^{6}$ Por ello, algunos autores llaman "rururbanas" a las poblaciones alteñas (Gilabert y Camarena, 2004:181-230).

4 Del total de 6752,113 habitantes que hay en el Estado de Jalisco, 17,291 viven en Villa Hidalgo. INEGI, II Conteo de población y vivienda 2005.

5 Gilabert y Camarena sostienen que Villa Hidalgo se ha desarrollado "más de los que sus recursos permitirían" gracias a la influencia espacial que sobre él ejerce Aguascalientes (Gilabert y Camarena, 2004:204).

6 Actualmente, de las 51,093 hectáreas de superficie del municipio, 25,400 son utilizadas para fines agrícolas; 20,224 se destinan a la actividad pecuaria; 2000 son de uso forestal y sólo 230 son de suelo urbano (del resto no se especifica el uso). En cuanto a la tenencia de la tierra, 46,490 hectáreas son privadas y 3,170 ejidales. No existe propiedad comunal (cfr. Plan Municipal de Desarrollo 2007-2009). 
La zona donde se encuentra Villa Hidalgo, conocida como los Altos de Jalisco, ${ }^{7}$ al igual que otras del país, "ha estado asociada desde siempre con la producción de ropa en el nivel de lo familiar. Particularmente, se ha considerado siempre que las mujeres de la región tienen una habilidad especial, heredada y enseñada por generaciones, para coser y bordar, lo que ha dado prestigio internacional a técnicas como el deshilado, el bordado y los tejidos, con los cuales se hacen productos como ropa de niño, vestidos, suéteres y blancos, entre otros" (CNIV, 2000:38). Así lo recuerda una villahidalguense:

"las muchachas (...) nos poníamos en la calle (...) y cada quien arrimaba una sillita de esas bajitas y nos poníamos a tejer, a bordar, a hacer punto de cruz, era en lo que se entretenían las muchachas de antes, porque ni nos dejaban venir a Aguascalientes a trabajar, ni trabajábamos en lo que no fuera la casa (...) Y pasaba gente que iba de fuera y nos preguntaba: iaquí dan clases de bordado, de tejido? No.

A nosotras nos enseñaban las mamás y las Madres, porque estuvimos en el colegio de las Maestras Católicas del Sagrado Corazón de Jesús (...) pues por eso se enseñaba uno a hacer de todo, y como no había más qué hacer, pues haciamos colchas, manteles, suéteres para nosotras. para la familia... (...)

Después hubo señoras que aprovechando el pueblo, que no había nada qué hacer (...). llevaban chalinas a tejer a mano, a las señoras, y las tejian y eso servía de aletilla, eso que tejiamos las niñas, y eso pues era una diversión para nosotros. Después (...) hubo gente que venía aquí a Aguascalientes y (...) llevaban las blusas para bordar a mano" (A4).

De hecho, desde hace dos décadas la zona occidental de México ha sido identificada por los estudiosos del desarrollo regional como una especie de "triángulo que se inicia en Aguascalientes, bordea hacia el sur por los Altos de Jalisco hasta Guadalajara, se desplaza al

7 Villa Hidalgo, uno de los 125 municipios del Estado de Jalisco, pertenece a la región denominada "Altos Norte", tradicionalmente conocida como "Los Altos de Jalisco", la cual, desde 1998, quedó dividida en la zona norte y la sur. "En 1998, por acuerdo del Ejecutivo Estatal, se estableció una nueva regionalización administrativa con el fin de armonizar el trabajo de las instituciones y dependencias públicas, potenciando el impacto regional de sus politicas y programas de acción. Fsta estrategia culminó con la definición de doce regiones, que permiten optimizar el impacto y la presencia regional de los programas públicos, garantizando una más eficiente utilización de los recursos presupuestales, una distribución equitativa del gasto gubernamental y un mejor aprovechamiento de la infraestructura disponible". http://www.jalisco.gob.mx/ nuestroedo/Municipios/index.html 
este hasta la pequeña y afamada ciudad manufacturera y comercial de Moroleón en el sur de Guanajuato y sube por el noroeste, de nuevo hacia Aguascalientes" (Arias, 1988:539, cit. en García, 2003).

Dentro de este "triángulo", cada pueblo se ha especializado en la elaboración de algún tipo de prenda

\footnotetext{
"Todos tienen mucho éxito, por ejemplo, San Miguel el Alto se define mucho por lo que es vestido de niña, la ropa de niña muy adornada, muy elaborada, y aparte están las fábricas de pants. Ayotlán se distingue mucho porque hace conjuntos, ropa para dama, para señora, entonces se enfocan mucho a lo que es la ropa de señora ya... ya no muy joven, y Zapotlanejo es ropa juvenil en mezclilla, y aquí es tejido, tejido y ya de todo, ya hay de todo, hay quienes hacen confección también, en mezclilla y de todo (...)

Y nos complementamos los pueblos, por ejemplo: nosotros le compramos mucha ropa a Zapotlanejo, y Zapotlanejo nos compra muchísima ropa a Villa Hidalgo, ¿por qué?, porque en Zapotlanejo no hay tejido y aquí le compramos lo que ellos hacen porque aquí la gente no hace lo mismo, y ya le compran a Moroleón y Moroleón les compra, entonces es un proceso natural, porque la gente ya acude naturalmente a estos centros de distribución" (DF6).
}

Villa Hidalgo se ha posicionado, desde la década de 1970, como centro de fabricación y comercio de prendas de vestir orientado a un mercado local y regional de ventas al mayoreo y menudeo. La mayoría de las fábricas del lugar producen ropa de tejido de punto para mujeres, y los comerciantes distribuyen, además de los productos locales, ropa de tela plana proveniente de otras ciudades. El mercado al que se dirige es al que demanda ropa casual moderna, barata y de calidad. No es un centro de desarrollo de moda (no existen marcas que se caractericen por sus diseños de vanguardia).

A continuación se expondrá la historia del desarrollo de la industria del vestido en Villa Hidalgo, la cual fue dividida en varias etapas, para conseguir mostrarla con claridad. 
EL CASO DE LA PRODUCCIÓN INDUSTRIAL DE PRENDAS DE VESTIR EN VIILA HidALGO, JALISCO ${ }^{8}$

Se podría considerar como primera etapa, la de los inicios de la industria del vestido en Villa Hidalgo, comprendida entre los años que van de 1940 a 1960.

En la década de los cuarentas, se producían pequeñas cantidades de cobijas y sarapes. Esta tarea generalmente era realizada por mujeres en grandes telares de bastidores de madera con clavos. La mercancía era comercializada por los hombres en distintas ciudades del país, principalmente el Distrito Federal, Guadalajara y Aguascalientes.

Una década después se comenzaron a utilizar las llamadas "máquinas de regla" con las que se tejían y confeccionaban las populares chalinas o "mañanitas" de tres picos, que posteriormente eran decoradas con bordado a mano y un fleco. Estas chalinas, distribuidas fundamentalmente en Aguascalientes, dieron una gran fama a Villa Hidalgo (cfr.CNIV, 2000).

Ese tradicional origen es conocido por quienes han crecido en el pueblo, y de esa manera lo narran:

"Más o menos empezó como en el cincuenta, pero eran tejidos muy artesanales, al principio eran señoras que en sus casas trabajan con lo que podian y hacian chalinas, cosas así, pero muy bonitas, muy elaboradas, y eso le empezó a dar la pauta a Villa Hidalgo, que la gente venía a comprarle a esas señoras y fue conde se inició esto, ya tiene bastantes años aquí en Villa Hidalgo, yo creo que como sesenta años debe tener ya la fabricación de ropa aquí en Villa Hidalgo" (DF2).

Las primeras máquinas manuales, remalladoras motorizadas y "rectas" se emplearon hasta los años sesentas, que fue cuando los hermanos Chávez las introdujeron

Los datos históricos aquí recogidos se obtuvieron del documento publicado en el año 2000 por la Cámara Nacional de la Industria del Vestido (CNIV), Delegación Villa Hidalgo, titulado: "Tejiendo la historia". También se incluyen datos del SIEM: Sistema de Información Empresarial Mexicano y de las entrevistas con fabricantes y comerciantes del pueblo. 
"se vinieron unos que son de Villa Hidalgo, pero que vivian en México, los de apellidos Chávez. Luego los Chávez trajeron mucha maquinaria de México, pero no había conocimientos en Villa Hidalgo, porque la gente estaba en ceros con respecto a lo que es la industria del tejido.

Fue como en el 64, 65, más o menos, cuando empezó la luz y, fue cuando llegó primero Dolores Díaz, José Delgado, los Chávez... y los Chávez llevaron gente ya capacitada..." (A4)

Con esas máquinas se montaron los primeros talleres "en forma" con una producción más elevada, que consistía en trajecitos de bebé, gorritos y chambritas, y más tarde las prendas que darían tanta relevancia a la industria, como la famosa "blusa pintor" para dama. Las máquinas que se utilizaban con mayor frecuencia eran manuales (cfr. CINV, 2000).

La segunda etapa de la historia de la producción de ropa sería la de los años setentas, en la que, a pesar de que Villa Hidalgo todavía era un pueblo en el que vivían "nomás del puro campo, del campo y poquito ganado, no había mucho, pero pues era un pueblo pobre" (DF9), paulatinamente se fueron introduciendo máquinas motorizadas, semiautomáticas y automáticas que permitieron un acelerado crecimiento de la producción.

\footnotetext{
"En ese entonces yo tenía clientes de León, de Zacatecas, de Guadalajara, de Aguascalientes, de San Luis, aquí, del centro, y pues, no era más que tenía unas maquinitas, primero eran manuales, después motoricé una y pues se sacaba poquita producción. Con tres, cuatro clientes que yo tenía, venían y se llevaban que... dos docenas cada quien y se iba vendiendo lo que salía. En ese entonces estaba bien porque todo lo vendía uno de contado, sacaba uno como cien, doscientas prendas a la semana y lo vendía uno de contado. Y después empezó a crecer lo de las tiendas y empezó uno a surtirle a las tiendas, pues son puros conocidos los que tienen las tiendas, pues la mayoría somos de aquí, conocidos, entonces uno les entregaba y hasta la actualidad, yo siempre sigo vendiendo aquí y parte de fuera, pero personas que vienen aquí" (DF9).
}

En aquellos años hubo también una adquisición masiva de máquinas familiares del tipo Singer y Brother, que facilitaron el surgimiento de varias microempresas familiares (CNIV, 2000).

Hasta ese momento "los talleres eran chiquitos y con máquinas muy manuales. La confección de las prendas con máquinas rectas u over pues no se daba, porque no había todavía electricidad, o sea, 
lo que marcó la pauta fue la electricidad. Y el impacto también fue el acercamiento con un mercado fuerte: Aguascalientes, San Luis y Zacatecas" (A2).

Con los primeros talleres aparecieron también las tiendas, como narra un comerciante, miembro de una de las familias pioneras en la venta de ropa:

“Mi mamá tenía fábrica, empezó a fabricar como en 1970, más o menos. En 1979, mis hermanos, que trabajaban en la fábrica de mi mamá, acordaron poner una tienda con mi cuñado. En aquel tiempo nada más había como tres tiendas de ropa, muy pequeñitas, en el Parián. La primera tienda grande que hubo fue la de mis hermanos, se llamaba DISOLO, de Guadalupe Soto, Dolores Díaz y Benjamín López.

Primero vendíamos sólo tejido, pero luego empezamos a traer ropa confeccionada de Ayotlán, de Arandas, de Guadalajara, Zapotlanejo, La Piedad, porque cada vez venía más gente y no sólo quería tejido, también querían otro tipo de ropa. Mi mamá también le creció a su fábrica, porque teníamos necesidad de más producto y pues llegó un momento en que ya era un poco complicado, un poco difícil atender los dos negocios, porque aunque éramos varios de familia, había bastante trabajo, hasta teníamos siempre fila en la calle. Empezamos a traer mercancía de México, de Irapuato, y fue creciendo hasta que llegó a ser una tienda grande. De ahí empezó a haber más tiendas, pequeñas, pero empezaron a crecer también, porque con la gente que venia, pues había gente para todos" (DT3).

De esa manera, el pueblo se fue dando a conocer

"Cuando se inició la industria (...) se traía mucho suéter de México para venderse aquí, aparte de lo que se fabricaba. Entonces se empezó a fabricar aquí y la gente venía y era muy barato el suéter; entonces, sin ninguna publicidad, o sea, cero publicidad, la publicidad la iba dando la gente que compraba un suéter: ¿dónde lo compraste?, pues en Villa Hidalgo. Entonces así fue como empezó a darse la publicidad de Villa Hidalgo, la que era de las mismas personas que compraban..." (DF6).

"En aquél entonces, cuando empezó esto a crecer, el pueblo se hizo de un prestigio; o sea, Villa Hidalgo tuvo un prestigio de calidad, de costura bien terminada y empezó la gente a venir" (DF9).

La explicación que los actuales empresarios dan de este desarrollo industrial y comercial tiene que ver con "condiciones sociales de confianza, reciprocidad y sentido de solidaridad social producto de la cultura de los villahidalguenses" (CNIV, 2000:33-34). Según la 


\title{
Cámara de la Industria del Vestido, en Villa Hidalgo:
}

\begin{abstract}
"El surgimiento de empresas familiares que crecieron de manera exponencial, debe mucho a la colaboración y la subcontratación que comenzaron a impulsar con empresas pequeñas de la región, y que atrajo de manera definitiva a diversos poblados como Calvillo, Teocaltiche y Villa Hidalgo, para que se insertaran en la dinámica de la producción de ropa. Lo mismo ocurrió con otras poblaciones ahora altamente reconocidas, como Moroleón en Guanajuato o Zapotlanejo en Jalisco.

Las historias de éxito se difundian con gran rapidez y comenzaron a multiplicarse. Las relaciones de cercanía y confianza social dieron como resultado que muchos patrones alentaran a sus trabajadores a instalarse por cuenta propia, llegando al grado de prestarles maquinaria -o vendérselas muy barata-y facilitarles insumos para que pudieran independizarse: todo ello basados en una promesa de pago que generalmente requería como único aval la palabra de honor del destinatario de los beneficios" (Ibid).
\end{abstract}

Un caso que ejemplifica lo anterior es el de un fabricante que comenzó a trabajar en 1976:

"Primero trabajaba tejiendo, como obrero, luego me puse a vender ropa, de los mismos con los que trabajaba, me facilitaban ropa para vender y me fue muy bien, y luego ya compré la máquina.

Desde un inicio cuando empecé ya tenía unas cuatro gentes, después me casé y ya empezamos a coser un poquito más.

Esa primera fábrica la tenía en la casa de mis papás, ahí me prestaron un cuarto. De ahí rentamos una casa, y luego ya nos fuimos a la casa propia, donde está la tienda, ahí teníamos la fábrica, y luego ya no cupimos y nos vinimos aquí (al Parque Industrial)" (DF6).

El ejemplo citado abre la posibilidad de pensar que la movilidad social pudo haber impulsado a otros a trabajar en el negocio de la ropa. ${ }^{9}$ De acuerdo con estudios realizados con respecto al nivel de "apertura" de la sociedad a la movilidad, se ha encontrado que la movilidad ascendente es más frecuente que la descendente y

\footnotetext{
"La expresión Movilidad Social se refiere al movimiento de los individuos y grupos entre las distintas posiciones socioeconómicas. La movilidad vertical es un desplazamiento hacia arriba o hacia abajo en la escala socioeconómica. De quienes ganan propiedades, renta o posición se dice que se mueven hacia arriba, mientras que los que las pierden se mueven hacia abajo (...) Por otra parte, se puede analizar en qué medida los hijos tiene el mismo tipo de empleo que sus padres o abuelos. Este tipo de movilidad se llama movilidad intergeneracional" (Giddens, 2000:344).
} 
se concentra mayoritariamente en los niveles intermedios de la estructura de clases (Giddens, 2000:349).

Otra consideración importante en este sentido es que la posibilidad de ascender en la escala socioeconómica tiene mucho qué ver con el aumento de los empleos en niveles superiores, aunque eso no quiere decir que también hayan aumentado las oportunidades de acceder a ellos (Íbid:350).

En el caso de Villa Hidalgo, el asenso no se ha dado de un puesto inferior a uno superior dentro de una misma empresa, sino de ser empleado a ser dueño, lo cual es visto como una tradición que se ha ido transmitiendo, de manera que el negocio de la ropa se percibe como una fuente "natural" de trabajo, tanto para quienes no tienen antecedentes familiares en la manufactura (como es el caso de ejemplo anterior), como para quienes sí los tienen:

\footnotetext{
"Empecé trabajando con mi mamá, con mi papá, ellos también tenían una fábrica de tejido, mis abuelos también, allá por los años... mil novecientos cuarenta y tantos ya trabajaban. Fra otro tipo de maquinaria, otro tipo de productos, hacian muchos sarapes, gabanes, todo ese tipo de ropa. Eran otros materiales, otro tipo de maquinaria, pero pues ya estamos enrolados, ahora sí que nacimos entre la ropa" (DF3)
}

El crecimiento de la actividad manufacturera de los años setentas continuó y tuvo su auge en las décadas posteriores, que podrían constituir la tercera etapa, la de los años ochentas y noventas

\footnotetext{
"En ese tiempo, gracias a Dios, lo poquito que producíamos, aquí mismo en Villa Hidalgo se vendía, e incluso nosotros no teníamos que salir y dar un paso para vender nuestro producto porque ahí llegaba la gente a la misma casa a levantarnos el pedido" (DF4).
}

Según el testimonio de productores de aquellos años, la demanda de mercancía era tal, que muchos talleres no se daban abasto

\footnotetext{
"Anteriormente, te estoy hablando todavía de cinco, seis, siete, ocho años atrás, yo no paraba ocho días al año, lo mismo para mí era septiembre que febrero que diciembre. Aquí la temporada fuerte es octubre, noviembre y diciembre, tres meses nos daban para comer al año (...) Yo no paraba ocho días, si acaso en agosto para dar limpieza a la maquinaria, mantenimiento, que
} 
me lo checaban, y me podía el alma porque se me acababa lo de bodega, y yo (necesitaba) para cumplirle al cliente" (DFl).

Al mismo tiempo, también a partir de la década de 1980, el comercio experimentó un gran dinamismo, porque se comenzó a vender localmente la producción que antes se distribuía en ciudades relativamente cercanas como Aguascalientes, San Juan de los Lagos y Guadalajara, y en otras como Tijuana, el Distrito Federal, Torreón o Mazatlán (CNIV, 2000:29-30).

"Aquí en el pueblo todo el crecimiento del comercio, se puede decir que fue del ochenta en adelante. Al principio, cuando yo me enseñe, ${ }^{10}$ había unos cinco, seis talleres, después, cuando ya llegué de Estados Unidos había unos... veinte, treinta; después llegó a... no sé exacto, ya en el ochenta, ochenta y cinco, ha de haber unos ciento y pico talleres y ahorita pues hay muchos talleres y pues muchísimo del comercio creció de la venta, de tiendas de ropa. Aquí indudablemente es la fuente de trabajo la industria y el comercio (...) toda la entrada es pura tienda, el centro y desde la entrada es pura tienda" (DF9).

Un momento importante en el crecimiento de la industria del vestido en Villa Hidalgo fue la construcción del Centro Comercial "Villa Textil", en octubre de 1987, como resultado de la unión de más de 60 comerciantes e industriales de la localidad (CNIV, 2000) quienes, al ver el número de clientes iba en aumento, decidieron iniciar el nuevo negocio. Así lo narra uno de los socios fundadores:

"Como en el ochenta y cuatro, ochenta y tres, ochenta y dos, esos años es cuando empezó a tener mucha afluencia la calle de Nieto (en Aguascalientes) y empezó a venir más gente de ahí a comprar. Por la central camionera (de Aguascalientes) también había varias tiendas y todos venían y compraban bastante, teníamos ahí muy buenos, excelentes clientes. Y pues de esa manera fue creciendo.

En momentos, aquí en Villa Hidalgo se concentró todo (el comercio) en el área de la iglesia y después empezó a crecer por el centro de salud. Después pusieron la Terminal por el centro de salud, pero se puso un poco complicado y la cambiaron acá a la Venustiano Carranza, entonces pues se empezó a ir gente y empezó a crecer toda esa calle. Luego ya era insuficiente toda el área para el estacionamiento de los autobuses, los carros, el paso de la gente, los autobuses especiales, porque para esa época, ochenta y cinco, ochenta y seis pues ya empezaron a venir

10 De 1969 a 1972 trabajó como tejedor en un taller. En 1972 se fue a trabajar a Estados Unidos en una fábrica de cerámica, hasta 1975, que regresó a Villa Hidalgo y empezó su propio taller. 
los autobuses especiales del norte y pues no era suficiente esa área.

Entonces, como en el ochenta y siete, ochenta y nueve comenzó a gestarse Villa Textil. Terminó de construirse en el noventa y uno; se abrió en el noventa y dos y se hizo la Terminal allá.

Yo formo parte del grupo, de la sociedad anónima que hizo la Terminal. La hicimos en nuestro terreno y ya después la regalamos, la cedimos al Municipio.

Yo creo que la gente, los autobuses estaban viniendo, entonces consideramos que era un buen momento para invertir iverdad? Hubo gente que tuvo miedo, por ejemplo: se ofrecía un terreno y decían: no, es demasiado caro, y al rato llegaba otra gente más lista y lo compraba y se ponía y le iba muy bien, y esa gente pues se daba de golpes. Y hubo otros que desde el principio vimos unos lugares, fuimos invirtiendo primeramente en terrenos, en construcciones y después en poner tiendas y bueno, pues no nos podemos quejar..." (DT3).

\section{En aquellos años la mayoría de los clientes provenían del norte del país:}

“Lo que era Nuevo León, Chihuahua y Coahuila, básicamente de esos tres estados y Zacatecas empezaba a venir mucha gente. Todavía no era muy conocido aquí, desde luego la gente que venía decía: no pues vamos al ranchito..." (DT3).

También a finales de los ochentas, un inversionista comenzó a construir el Centro Comercial "Las Palmas", ubicado enfrente de "Villa Textil" (cfr.A5).

\footnotetext{
"Ahí es un solo propietario, originalmente eran cuarenta locales que estaban enfrente, él tuvo la idea de construir atrás y dijo: bueno, voy a construir atrás, aquí está la maqueta iquién quiere locales? Y ya uno iba y decía: bueno, pues yo quiero este, yo quiero este, perfecto. La renta es de tanto, tú me vas a pagar tres años por adelantado y yo en un año te lo voy a entregar. Y se le rentaron todos así, en realidad lo que pasó es que todos pagaron la construcción del centro comercial para después seguir pagando la renta de lo que son los locales. Pero eso se daba porque había mucha demanda de locales, era muy atractivo el negocio en Villa Hidalgo, era muy rentable un negocio en Villa Hidalgo" (DT2).

Aquella fue la época más floreciente del pueblo, a partir de la cual creció su fama y el número de pequeños empresarios que acudían ahí en busca de fortuna. Uno de ellos, originario del Distrito Federal, explica cómo eran esos años:
}

"Villa Hidalgo se convirtió en el polo de atracción de la comarca en sí, incluido Aguascalientes y sí, en efecto, atrajo a muchísimos a buscar aquí trabajo y lo encontraron, a buscar fortuna y la 
encontraron, era tierra de promisión Villa Hidalgo, se convirtió en una tierra de promisión, y además, de satisfacción de esperanzas" (DT6).

Muchas de las personas que iniciaron sus negocios en esos años, crecieron rápidamente

\footnotetext{
"Nuestras tiendas nacieron como negocio familiar, lo creamos mi papá y yo, cuando yo estaba soltero. Empezó a irnos muy bien, invitamos a mi hermana, luego invitamos a mi otro hermano, luego invitamos a un primo y fue creciendo, llegamos a tener seis sucursales, una en Zapotlanejo y las demás aquí. Pero llegó el momento en donde nos decía nuestro primo que estaba en Zapotlanejo: mándanos mercancía, y yo le tenía que decir: es que no tengo, toda la mercancía que llega se vende, entonces ya no te puedo mandar. Entonces eran otros momentos ino?" (DT2).
}

Finalmente, fue a principios de 1990 cuando la oferta de ropa comenzó a diversificarse con la introducción de confección de tela plana, que se sumó a la de tejido de punto. Asimismo, la localidad resultaba ya un polo de atracción importante para que se instalaran comerciantes procedentes de otros lugares de la república (CNIV, 2000), de manera que ya en el año 1997 se tiene registrada en la Cámara de Comercio de Guadalajara, la apertura de 75 nuevas tiendas, número que se irá incrementando cada año: en 1998 abren 85 nuevos negocios, en 1999, 94. El año 2000 tuvo 123 registros (SIEM, 2007), de modo que, en total, podrían llegar a ser hasta hoy, alrededor de 1,500 (cerca de una tienda por cada 10 habitantes).

Villa Hidalgo, Jalisco

\begin{tabular}{lllll}
\hline & 1997 & 1998 & 1999 & 2000 \\
Nuevos registros de tiendas & 75 & 85 & 94 & 123 \\
Fuente: Sistema Estatal de Información Jalisco & & & \\
\hline
\end{tabular}

La cuarta etapa de la historia de la industria del vestido en Villa Hidalgo es la contemporánea, iniciada en la primera década del tercer milenio, e incluida la crisis económica del año 2008. 
En el año 2000 comenzó a funcionar el "Parque Industrial Villa Hidalgo" y el padrón de licencias municipales "contabilizaba más de 350 fábricas de ropa (lo que significaría aproximadamente una fábrica por cada 44 habitantes). José Carmen Villalpando, pionero de la industria del vestido en Villa Hidalgo, asegura que el número de empresas domiciliarias podría ser similar al de las registradas oficialmente, lo que se traduciría en al menos 700 talleres de la confección" (García, 2003:83).

Según datos del INEGI, en 2004 estaban registradas 630 tiendas de ropa al por menor, que ocupaban a 1,609 personas (frente a 1,170 en 1999, sin número de tiendas especificado). Los establecimientos que fabricaban ropa en 1999 eran 145, y en el 2004 se redujeron a 49. Sin embargo, el personal ocupado el primer año era 372 y en 2004, ascendió a 820 personas."

Villa Hidalgo, Jalisco

\begin{tabular}{lllll}
\hline & No. de talleres & Personal ocupado & No. de tiendas & Personal ocupado \\
\hline 1999 & 145 & 372 & & 1,170 \\
2004 & 49 & 820 & 630 & 1,609 \\
\hline
\end{tabular}

Fuente: INEGI, Censos Económicos 1999 y 2004.

Sin embargo, en la actualidad, la situación "ha cambiado mucho: la gente que compra para vender ya es la minoría, ya es el 10\%, 12\%, y el ochenta y tantos ya es gente que compra para uso final, o sea, viene de Aguascalientes, de San Luis, Zacatecas, a comprar para toda la familia. Porque ya para la que venía de Chihuahua (...) ya no le resulta tan buen negocio" (DT2).

Estas nuevas circunstancias son resultado de la crisis de 2008, la cual ha sido perjudicial, debido principalmente a las importaciones de ropa china, a raíz de las cuales, "lo que es el textil, el calzado, se

FUENTE: SEIJAL (Sistema Estatal de Información Jalisco), con base en datos proporcionados por los Censos Económicos 1999 y 2004 del INEGI. http:/sig.jalisco.gob.mx/cedulas/ 
fue para abajo" (DF3), porque esa ropa "no paga un solo peso de impuestos, nada, y eso nos afecta mucho" (DF2).

Según datos de la Cámara Nacional de la Industria del Vestido (CNIV), todos los días entran a México prendas de vestir fabricadas en China, a precios con los que es muy difícil competir: blusas de 1.41 pesos, pantalones de 1.45 y vestidos de 2.84 pesos, por lo que "algunos (fabricantes mexicanos) han tenido que cerrar plantas" (CNIV, 2010b).

Una de las consecuencias que ha traído consigo esta competencia ha sido que las condiciones de los negocios de Villa Hidalgo hayan tenido que cambiar

\footnotetext{
"Antes uno no arriesgaba demasiado, decía: voy a cortar esta prenda, voy a cortar mil, a ver qué pasa, y no pasaba nada, se vendia. Ahorita los cortes son 200, 150, diez por color, porque ya no puede uno arriesgar tanto.
}

La producción ha venido bajando, la manufactura está bastante mal, sobre todo lo original de Villa Hidalgo. Yo creo que es una de las crisis más fuertes: la industria textil tiene un problema serio este año (2008) (...) Ahorita la gente ya empieza a cambiar un poquito, poquito, ya a lo mejor en lugar de que todo junto, te voy a dar treinta, sesenta o noventa días para que me pagues la renta, a lo mejor los últimos tres años no ha cambiado la renta, o sea, se ha venido acomodando" (DT2).

La situación antes mencionada ha contribuido a que cambie la percepción de Villa Hidalgo como "tierra de promisión"

"Siento que todo mundo fuera de Villa Hidalgo, cree que Villa Hidalgo es un lugar donde se hace un montón de dinero, donde se hace un dineral. En realidad, se gana más dinero sólo en la temporada alta, que dura cinco o seis semanas cada año. Han bajado las ventas porque ha aumentado la competencia. Por eso tienen que bajar sus costos" (EFCl).

Los empleados también son conscientes de la situación por la que atraviesa Villa Hidalgo, "pues es un año que está difícil" (EFC6).

"Ahorita yo pienso que está muy mal. Otros años nosotros trabajábamos mayo, junio, julio bien; 
ahorita nos da trabajo un mes, pero no es suficiente, porque con cincuenta prendas que tú hagas a la semana no te mantienes, está demasiado bajo. Hasta que se venga la temporada, supuestamente, pero la temporada es pocas semanas" (EFC7).

Sin embargo, los villahidalguenses parecen ser conscientes de que la producción de prendas de vestir es una tradición consolidada y, por lo mismo, llamada a permanecer: "Yo creo que como el 95\% o un poco más nos dedicamos a fabricar o a comercializar ropa, pero todos estamos dedicados a la ropa" (DF2).

Existe, además, un cierto sentido de que la fabricación de ropa es la vocación del pueblo

"Simple y sencillamente se nos dio que Dios puso los ojos aquí en el pueblo y pues ya nosotros somos los que hemos hecho lo que siguió iverdad?" (DF4), porque "ni tenía tradición manufacturera, ni estaba bien comunicado (hace veinte años, para ir de Guadalajara a Aguascalientes se pasaba por Teocaltiche, no por Villa Hidalgo), ni hubo alguna persona clave o un grupo que impulsara esta actividad. Simplemente, por suerte que en Villa Hidalgo prendió lo de la ropa, por la carestía y todo, porque Dios es grande y nos quiere mucho" (DT2).

De acuerdo con lo anteriormente expuesto, el hecho de que el negocio de la ropa haya tenido la difusión y el éxito comercial de los años setentas hasta la actualidad, hace que Villa Hidalgo sea percibida por sus pobladores como localidad "históricamente manufacturera", debido a que posee "vocación textil".

Tener conciencia de ser un pueblo con vocación textil trae consigo que tanto los productores como los comerciantes tengan confianza en que podrán continuar dedicándose al negocio de la ropa, aunque para ello deban adoptar medidas de adaptación a las nuevas circunstancias económicas.

Al respecto, un grupo de empresarios ya ha conseguido poner en marcha una teñidora de hilo, de manera que "con esto nuevo de la teñidora, yo ya veo otro panorama muy diferente, porque vamos a abaratar mucho nuestros costos de antes, ya podemos competir, ahora sí, que nos echen a los chinos, ique son los que nos están matando!" (DF4). 
Losfabricantes tienen muchas esperanzas puestas en esa empresa, según lo expresan ellos mismos: "le tenemos mucha fe, porque Villa Hidalgo es de lo que vive, entonces nosotros vamos a proveerle a los de Villa Hidalgo de algo que ocupan día con día, todos, entonces le vemos muchas, pero muchas posibilidades a este proyecto" (DF4).

Aunado a lo anterior, existen todavía algunos retos a enfrentar, como la innovación y la búsqueda de nuevos mercados, estrategias necesarias para estar al día, pendientes de las necesidades de los clientes. No obstante, el balance de los villahidalguenses con respecto al futuro del negocio de la ropa es positivo, por varios motivos. En primer lugar, porque los empresarios que se instalaron en las décadas pasadas tuvieron la oportunidad de consolidar sus negocios, de manera que ahora que ha cambiado la perspectiva económica, cuentan con recursos para hacerle frente

"La gente que ahora está bien económicamente es porque hace años hizo dinero y ahora viven de sus rentas. Si ahorita alguien se pone, dudo que aguante. Nosotros es el primer año que descansamos a los empleados" (DF8).

Entre los recursos para salir adelante se encuentran la confianza que tienen en su propio trabajo y el buen potencial de comercialización del producto que elaboran

\footnotetext{
"Tenemos los cimientos muy bien plantados, y es que ya estamos impuestos: sabemos trabajar duro, sabemos movernos. Nuestro producto tiene la capacidad de competir, tiene la calidad, tiene todo. Nomás nos falta salir a buscar más mercados" (DF8).
}

Además de contar con esos recursos, los villahidalguenses ven difícil perder su posicionamiento en el mercado

"Sí, sí va a seguir, porque el pueblo desde el principio creó un prestigio de ropa de buena calidad, y porque también tiene muy buenos precios (...) Entonces esto lo hace que siga existiendo esta fuente de trabajo" (DF9).

Es decir, los empresarios tienen confianza en que el negocio al que se dedican "es muy noble (...) No creo que la gente se vaya a 
dejar de vestir, no se puede, es un producto de primera necesidad, entonces no se puede terminar el negocio de la ropa" (DF3). Por otro lado, existe en algunas personas la convicción de que la crisis se superará, porque es sólo una etapa pasajera, como otras. Ello contribuye a tener una visión optimista ante las dificultades

\footnotetext{
"Yo de que recuerdo, van como unas tres crisis de estas, de que se detiene tanto que la gente no vemos por dónde salir, pero luego empieza a agarrar, empieza a agarrar..." (DF4). "Siempre hay ciclos. La ropa china va a terminar encareciéndose. Esto no se va a acabar, lo chino pasará y nosotros seguiremos" (A5).
}

Finalmente, también existe la seguridad de que "Villa Hidalgo es un pueblo bendito por Dios" (EFCl) y ello aporta una menor inquietud ante el futuro.

El breve recorrido por la historia industrial y comercial de Villa Hidalgo aquí expuesto muestra su rápida expansión y capacidad para ocupar personal. Llama la atención que a lo largo de sólo 40 años, el negocio de la ropa ha conseguido dotar a los villahidalguenses no sólo del sustento económico, sino también de una conciencia de que su vocación es el trabajo textil, la cual define al pueblo como "históricamente manufacturero". Esa conciencia contribuye a que la actual situación de crisis económica sea enfrentada con la esperanza de ser superada.

\section{BiBLIOGRAFIA}

- Botello Aceves, Brígida, Magdalena Heredia Mendoza y Raquel Moreno Pérez. Memoria del Municipio en Jalisco. Guadalajara, Jal. , UNED, 1987, en: Enciclopedia de los Municipios de México, 1988.

- Cámara Nacional de la Industria del Vestido, 2010b: "Ingresa a México ropa china a peso", en: Mural, Guadalajara, Jalisco, 9 de marzo de 2010.

- Cámara Nacional de la Industria del Vestido, Delegación Villa 
Hidalgo, 2000: Tejiendo la historia. Orígenes y fundadores de la industria del vestido en Villa Hidalgo, Jalisco, México.

- García, Alejandro, 2003: La importancia de las relaciones sociales de los empresarios en la constitución de redes de pequeñas y medianas empresas, Tesis de Maestría en Sociología Industrial y del Trabajo, Universidad Autónoma de Aguascalientes, México.

- Giddens, Anthony, 2000: Sociología, Alianza Editorial, Madrid, España.

- Gilabert, César y Margarita Camarena Luhrs, 2004: El alteño global, Universidad de Guadalajara, El Colegio de Jalisco, México.

- Sistema de Información Empresarial Mexicano (SIEM), www. siem.gob.mx. 